\title{
Micro-scaled Quantitative Method to Analyze Olive Oil Polyphenols
}

\section{Giovanni Caprioli, Maria Chiara}

\section{Boarelli, Massimo Ricciutelli, Gianni}

\section{Sagratini \& Dennis Fiorini}

Food Analytical Methods

ISSN 1936-9751

Food Anal. Methods

DOI 10.1007/s12161-019-01433-7

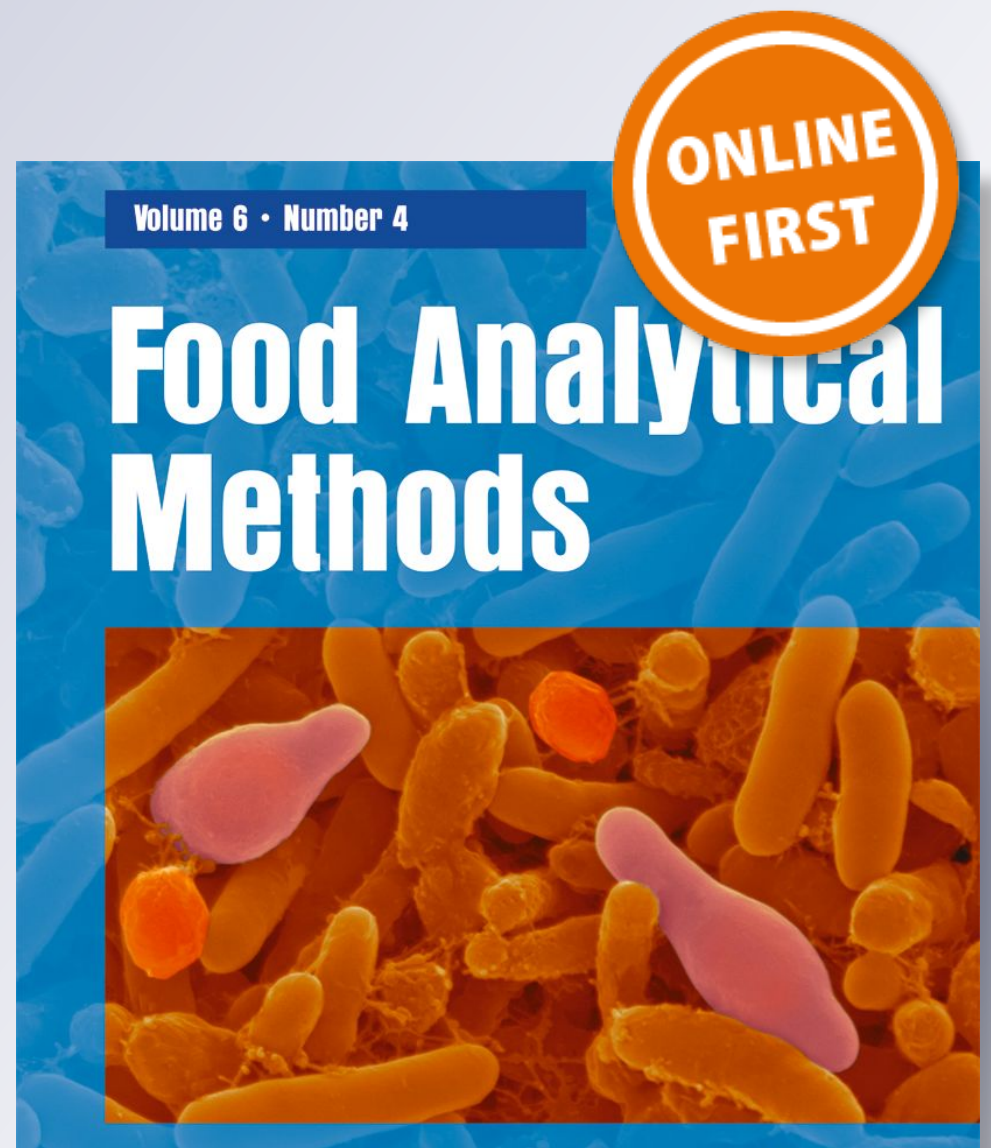

August 2013

Available + z: online

\section{包 Springer}


Your article is protected by copyright and all rights are held exclusively by Springer Science+Business Media, LLC, part of Springer Nature. This e-offprint is for personal use only and shall not be self-archived in electronic repositories. If you wish to selfarchive your article, please use the accepted manuscript version for posting on your own website. You may further deposit the accepted manuscript version in any repository, provided it is only made publicly available 12 months after official publication or later and provided acknowledgement is given to the original source of publication and a link is inserted to the published article on Springer's website. The link must be accompanied by the following text: "The final publication is available at link.springer.com". 


\title{
Micro-scaled Quantitative Method to Analyze Olive Oil Polyphenols
}

\author{
Giovanni Caprioli ${ }^{1} \cdot$ Maria Chiara Boarelli ${ }^{2} \cdot$ Massimo Ricciutelli $^{3} \cdot$ Gianni Sagratini $^{1} \cdot$ Dennis Fiorini $^{2}$ (I)
}

Received: 21 September 2018 / Accepted: 7 January 2019

(C) Springer Science+Business Media, LLC, part of Springer Nature 2019

\begin{abstract}
This study aims to improve an analytical method to quantify phenolic substances in olive oil. In order to minimize time required and quantity of solvents, sample extract preparation performed for a previously developed high-performance liquid chromatography-diode array detector to quantify olive oil polyphenols has been ten times downscaled and then validated. The new method performs the extraction of phenolic substances from $0.5 \mathrm{~g}$ of oil and allows to quantify the phenolic acids vanillic acid, $p$-coumaric acid, and ferulic acid; the phenolic alcohols tyrosol and hydroxytyrosol; secoiridoid derivatives; the lignans pinoresinol and acetoxypinoresinol; and the flavonoids luteolin and apigenin. Recoveries obtained were 66-89\% for phenolic alcohols, 64-90\% for phenolic acids, 93-96\% for oleuropein (used as a reference for secoiridoid derivatives), 71-95\% for flavonoids, and $97-100 \%$ for lignans. The total quantity of organic solvents used in the sample preparation is decreased from 30 to $3 \mathrm{~mL}$ with an important abatement of waste, costs, and working time requested.
\end{abstract}

Keywords Olive oil polyphenols $\cdot$ Quantitative determination $\cdot$ HPLC-DAD $\cdot$ Secoiridoid derivatives $\cdot$ Green method

\section{Introduction}

Olive oil phenolic substances have been recognized to have a key role in determining oil quality. Furthermore, the European Food Safety Authority (EFSA) has allowed the acknowledgement of a health claim on olive oil polyphenols. Thus, there is a great interest, both of the producer, of the consumer, and in organisms demanded to the official analytical control, in knowing the content of polyphenols found in an olive oil, and especially in the phenolic substances allowing the acknowledgement of the health claim, that are "hydroxytyrosol and its derivatives, e.g., oleuropein complex and tyrosol" (EU 2012). There are several methods available in literature to perform this analysis (e.g., Carrasco-Pancorbo et al. 2005; Franco et al. 2014; Capriotti et al. 2014; Flores et al. 2012; Suárez et al. 2008; Caporaso et al. 2015; Bayram et al. 2012; Bakhouche et al. 2013; Bendini et al. 2007) starting from

Dennis Fiorini

dennis.fiorini@unicam.it

1 School of Pharmacy, University of Camerino, V. S. Agostino 1, I-62032 Camerino, MC, Italy

2 School of Science and Technology, Chemistry Division, University of Camerino, V. S. Agostino 1, I-62032 Camerino, MC, Italy

3 HPLC-MS Laboratory, University of Camerino, V. S. Agostino 1, I-62032 Camerino, MC, Italy which we developed a new method allowing the analysis of these substances, and other minor phenolic substances, like phenolic acids, flavonoids, and lignans, obtaining some improvements mainly in terms of chromatographic separation (Ricciutelli et al. 2017), making use of a reverse-phase analytical column never used before in this application, the Synergi Polar $(4 \mu \mathrm{m}, 80 \AA, 250 \times 4.6 \mathrm{~mm})$. The application of this method to process a high number of samples led us to the idea of downscaling the quantities of sample and solvents used, in order to have an abatement first of all in terms of time requested to prepare the sample (the evaporation of the low volatile extraction solvents, water and methanol, is in fact a limiting factor), but also an improvement in terms of environmental impact, with reduced quantity of organic solvents used and reduced waste and overall analytical costs. Thus, the aims of the present study were (i) to downscale the previously developed method, (ii) to validate the new method, and (iii) to apply the new method to extra virgin olive oils (EVOOs) and olive oils (OOs).

\section{Materials and Methods}

\section{Reagents and Standards}

The analytical standards of hydroxytyrosol (CAS number 10597-60-1), tyrosol (CAS number 501-94-0), vanillic acid 
(CAS number 121-34-6), oleuropein (CAS number 32619-424), luteolin (CAS number 491-70-3), and apigenin (CAS number 520-36-5) were purchased from Extrasynthese (Genay, France). $p$-Coumaric acid (CAS number 501-98-4), ferulic acid (CAS number 537-98-4), and pinoresinol (CAS number 487-36-5) were purchased from Sigma-Aldrich (Milano, Italy). Syringic acid was purchased by Fluka (CAS number 530-57-4) and used as internal standard (IS). Standard stock solutions of each compound were prepared by dissolving $10 \mathrm{mg}$ of pure analytical standard in $10 \mathrm{~mL}$ of $\mathrm{MeOH}$. Standard working solutions, at various concentrations, were prepared when needed by appropriate methanol dilution of stock solution aliquots.

HPLC-grade methanol, hexane, and isopropanol were purchased from Sigma-Aldrich (Milano, Italy). Water (resistivity above $18 \mathrm{M} \Omega \mathrm{cm}$ ) was obtained from a Milli-Q SP Reagent Water System (Millipore, Bedford, MA, USA). All the solvents and solutions were filtered through a $0.45-\mu \mathrm{m}$ PTFE filter from Supelco (Bellefonte, PA, USA) before use.

\section{Sample Collection}

Ten EVOOs and ten OOs were purchased from a supermarket and stored at room temperature away from light until the analysis.

\section{Sample Extraction}

An amount of $0.5 \mathrm{~g}$ of oil was weighed in a $2-\mathrm{mL}$ vial with Teflon screw cap, the sample was dissolved in $0.5 \mathrm{~mL}$ of hexane, and a fixed aliquot of IS (i.e., $20 \mu \mathrm{L}$ of syringic acid methanolic solution at a concentration of $100 \mathrm{mg} \mathrm{L}^{-1}$ ) was added. The solution is extracted with $4 \times 0.5 \mathrm{~mL}$ of methanol: water $(60: 40, v / v)$ by means of a vortex device. After each extraction, samples were centrifuged ( $5 \mathrm{~min}, 5000 \mathrm{rpm}$ ) and the hydro-alcoholic solutions were collected in a 4-mL screw cap vial. Hexane $(1 \mathrm{~mL})$ was added to the final hydroalcoholic solution (in order to remove traces of acylglycerols left), vortexed and centrifuged for $5 \mathrm{~min}$ at $5000 \mathrm{rpm}$. The hexane phase is removed and the hydro-alcoholic solution was transferred into a $10-\mathrm{mL}$ round bottom neck flask and evaporated to dryness by means of a rotary evaporator under reduced pressure. The dried extract was reconstituted with $0.25 \mathrm{~mL}$ of HPLC-grade methanol, filtered through a $0.45-\mu \mathrm{m}$ PTFE filter, and collected into a 2-mL high recovery vial before HPLC-DAD-ESI/MS analysis.

\section{HPLC-DAD-MS Analysis}

Chromatographic analyses were performed by means of a high-performance liquid chromatograph coupled with a diode-array detector (DAD) and a mass spectrometer detector (ion trap) equipped with an electrospray ionization (ESI) source (Agilent 1100, Santa Clara, CA, USA), in the same conditions reported by Ricciutelli et al. (2017), using a chromatographic column a Synergi Polar $(250 \times 4.6 \mathrm{~mm}, 4 \mu \mathrm{m})$. The mobile phase was water with $0.1 \%$ formic acid (A) and methanol $/ i$-PrOH 90:10 $v / v$ with $0.1 \%$ formic acid (B) working in gradient mode at a flow rate of $1 \mathrm{~mL} \mathrm{~min}^{-1}$. The solvent composition varied as follows: $0 \mathrm{~min}, 30 \% \mathrm{~B} ; 0-40 \mathrm{~min}, 60 \%$ $\mathrm{B} ; 40-50 \min 95 \% \mathrm{~B}$; then the column was reconditioned. The column temperature was set at $35^{\circ} \mathrm{C}$ and the injection volume was $10 \mu \mathrm{l}$. HPLC-DAD analysis, that was used for the quantification, was performed monitoring different wavelengths: $260 \mathrm{~nm}$ for vanillic acid, $280 \mathrm{~nm}$ for tyrosol, hydroxytyrosol, secoiridoids derivatives (namely the dialdehydic form of decarboxymethyl elenolic acid linked to hydroxytyrosol or to tyrosol, an isomer of the oleuropein aglycon, and ligstroside aglycon), pinoresinol, acetoxypinoresinol, and syringic acid; $310 \mathrm{~nm}$ for $p$-coumaric acid, $325 \mathrm{~nm}$ for ferulic acid, $338 \mathrm{~nm}$ for apigenin, and $350 \mathrm{~nm}$ for luteolin. Quantification was performed as reported in the above mentioned study with the only exception of secoiridoid derivatives which were quantified using tyrosol calibration curve that resulted to provide very similar results as compared to the previously reported approach. In the present study, the mass spectrometer was used only for confirmation of the identity of the analytes and to identify the most abundant secoiridoid derivatives mentioned above.

\section{Method Validation}

The method was validated by determining linearity, repeatability, reproducibility, recovery at two fortification levels, limits of detection (LOD), and limits of quantification (LOQ). The main validation parameters obtained are reported in Table 1. For the assessment of the validation parameters related to the quantification of secoiridoid derivatives, oleuropein was used due the lack of their commercial availability and since it has a structure similar to secoiridoid derivatives found in higher amount in EVOO (especially those deriving from its hydrolysis).

Calibration curves of the analyzed compounds were constructed injecting $10 \mu \mathrm{l}$ of standard solutions at seven different concentrations (according to each analyte) in HPLC-DAD. Linearity was assessed for each species in the concentration range found in the samples and was very good, with a correlation coefficient within the range 0.9988-0.9999.

Repeatability and reproducibility were evaluated by analyzing both the extract from an EVOO sample and a standard mixture of polyphenols, three times within a day (repeatability) and for 3 days (reproducibility) and measured in terms of relative standard deviation percentage (RSD \%).

The recoveries were determined by spiking the olive samples with a standard mixture of polyphenols at two different levels, i.e., spike 1: $0.04 \mathrm{mg} \mathrm{kg}^{-1}$ (vanillic acid, $p$-coumaric 
Table 1 Method validation parameters

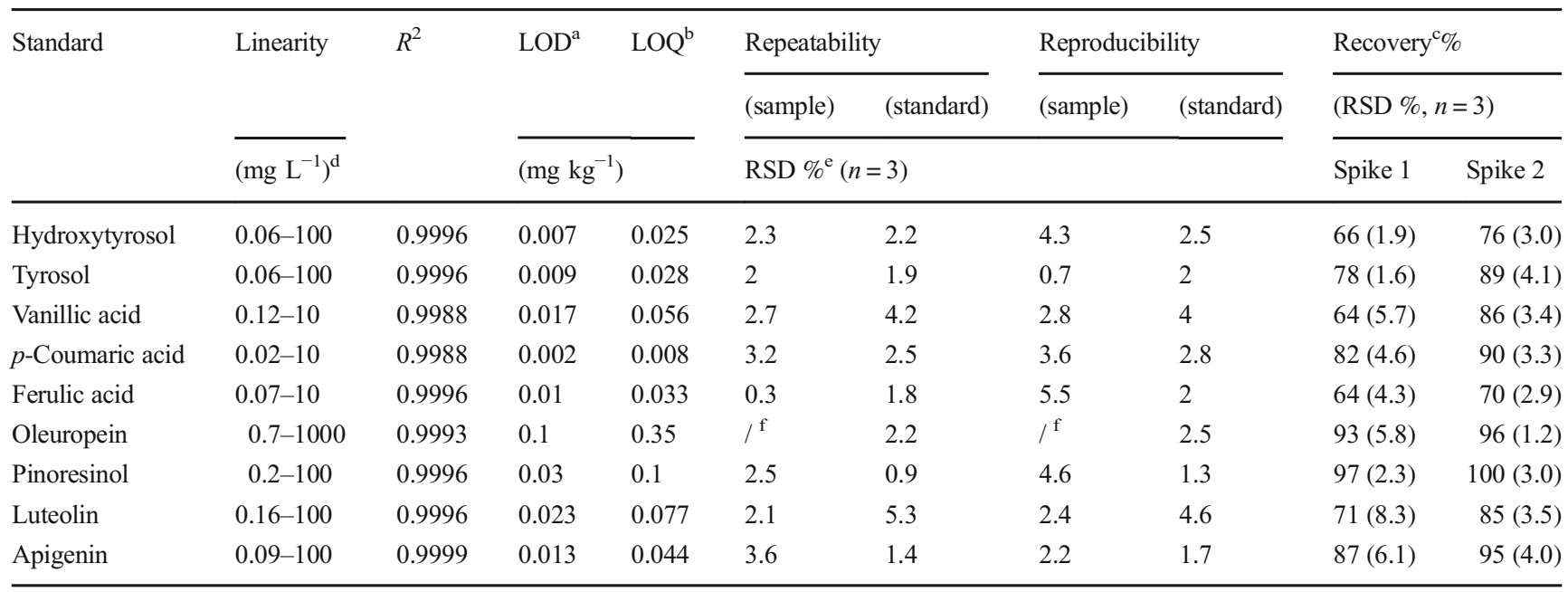

${ }^{\mathrm{a}} L O D$ limit of detection (expressed as mg of analyte in $\mathrm{kg}$ of oil), ${ }^{\mathrm{b}} \mathrm{LOQ}$ limit of quantification (expressed as mg of analyte in $\mathrm{kg}$ of oil), ${ }^{\mathrm{c}}$ determined by analyzing an olive oil sample spiked at two levels of concentrations; spike 1: $0.04 \mathrm{mg} \mathrm{kg}^{-1}$ (vanillic acid, $p$-coumaric acid, ferulic acid), $10 \mathrm{mg} \mathrm{kg}{ }^{-1}$ (hydroxytyrosol, tyrosol, luteolin, pinoresinol, apigenin), and $100 \mathrm{mg} \mathrm{kg}^{-1}$ (oleuropein); spike $2: 2 \mathrm{mg} \mathrm{kg}^{-1}$ (vanillic acid, $p$-coumaric acid, ferulic acid), $20 \mathrm{mg} \mathrm{kg}^{-1}$ (hydroxytyrosol, tyrosol, luteolin, pinoresinol, apigenin), and $200 \mathrm{mg} \mathrm{kg}^{-1}$ (oleuropein); ${ }^{\mathrm{d}} \mathrm{mg}$ of standard analyte in L of solution; ${ }^{\mathrm{e}} \mathrm{RSD}$ : relative standard deviation. ${ }^{\mathrm{f}}$ Oleuropein was not detected in the analyzed samples

acid, ferulic acid), $10 \mathrm{mg} \mathrm{kg}^{-1}$ (hydroxytyrosol, tyrosol, luteolin, pinoresinol, apigenin), and $100 \mathrm{mg} \mathrm{kg}^{-1}$ (oleuropein) and spike 2: $2 \mathrm{mg} \mathrm{kg}^{-1}$ (vanillic acid, $p$-coumaric acid, ferulic acid), $20 \mathrm{mg} \mathrm{kg}^{-1}$ (hydroxytyrosol, tyrosol, luteolin, pinoresinol, apigenin), and $200 \mathrm{mg} \mathrm{kg}^{-1}$ (oleuropein).

The repeatability of the method was also evaluated on the replicated analyses performed with the spiked samples used to assess recovery. The limits of detection and the limits of quantification of the different analytes were estimated on the basis of 3:1 and 10:1 signal to noise ratios.

\section{Results and Discussion}

The developed method applies to $0.5 \mathrm{~g}$ of EVOO that is dissolved in $0.5 \mathrm{~mL}$ of hexane and added with internal standard solution and then extracted four times, each with $0.5 \mathrm{~mL}$ of a solvent mixture methanol/water 6/4. The final volume of hydro-alcoholic extract is washed with $1 \mathrm{~mL}$ of hexane to remove triacylglycerols traces and finally dried and dissolved in $250 \mu \mathrm{l}$ of methanol. As compared to the method we previously developed (Ricciutelli et al. 2017), there is 10-fold decrease of the solvents and sample needed, leading to a great reduction of the time requested for sample preparation, that is largely due to the long time needed to evaporate the relatively low volatile solvent extraction mixture used (6/4 methanol/ water). Furthermore, in comparison to other methods reported in literature, where purification by solid phase extraction (SPE) (e.g., Franco et al. 2014; Capriotti et al. 2014) or acid/ alkaline hydrolysis (Carrasco-Pancorbo et al. 2005) is exploited, that is usually time-consuming steps, a further reduction of time is provided. Additionally, the method gives a substantial improvement in terms of environmental impact, with reduced quantity of organic solvents used and reduced waste. The common amount of oil used is higher with respect to our method (i.e., $0.5 \mathrm{~g}$ ), being in the range $1-10 \mathrm{~g}$ (Caporaso et al. 2015; Pirisi et al. 2000; De La Torre-Carbot et al. 2005; Selvaggini et al. 2006) as well as the corresponding solvent volume used for dissolving the oil, usually in the range 6-10 mL or more (Franco et al. 2014; Caporaso et al. 2015; Pirisi et al. 2000; De La Torre-Carbot et al. 2005; Selvaggini et al. 2006; Pirisi et al. 1997).

The amount of extraction solvent used in the present work ( $2 \mathrm{~mL}$ as total amount) is lower with respect to the solvent volumes used in other methods, which often are in the range 10-25 mL (Caporaso et al. 2015; Montedoro et al. 1992) or even more (about $40 \mathrm{~mL}$ ) if considering the total solvent amount used for the SPE process (Franco et al. 2014; De La Torre-Carbot et al. 2005).

Thus, the developed method aimed to extract and quantify polar-phenolic substances in olive oils with an important abatement of waste, costs, and working time requested for sample preparation.

In Fig. 1, a chromatogram obtained from the HPLC-DAD analysis of a standard mixture is reported and in Fig. 2, typical chromatograms obtained from the analysis of EVOO and OO extracts are reported. The most abundant secoiridoid derivatives found in EVOO (the dialdehydic form of decarboxymethyl elenolic acid linked to hydroxytyrosol or to tyrosol, an isomer of the oleuropein aglycon, and ligstroside aglycon) were quantified by using tyrosol as external standard as also done in other studies (Caporaso et al. 2015; IOC 2009) 
Fig. 1 HPLC-DAD

chromatogram $(\lambda=280 \mathrm{~nm})$ of a standard mixture composed by hydroxytyrosol, tyrosol, vanillic acid, syringic acid (internal standard), $p$-coumaric acid, ferulic acid, oleuropein, pinoresinol, luteolin, and apigenin, obtained by applying the optimized downscaled method

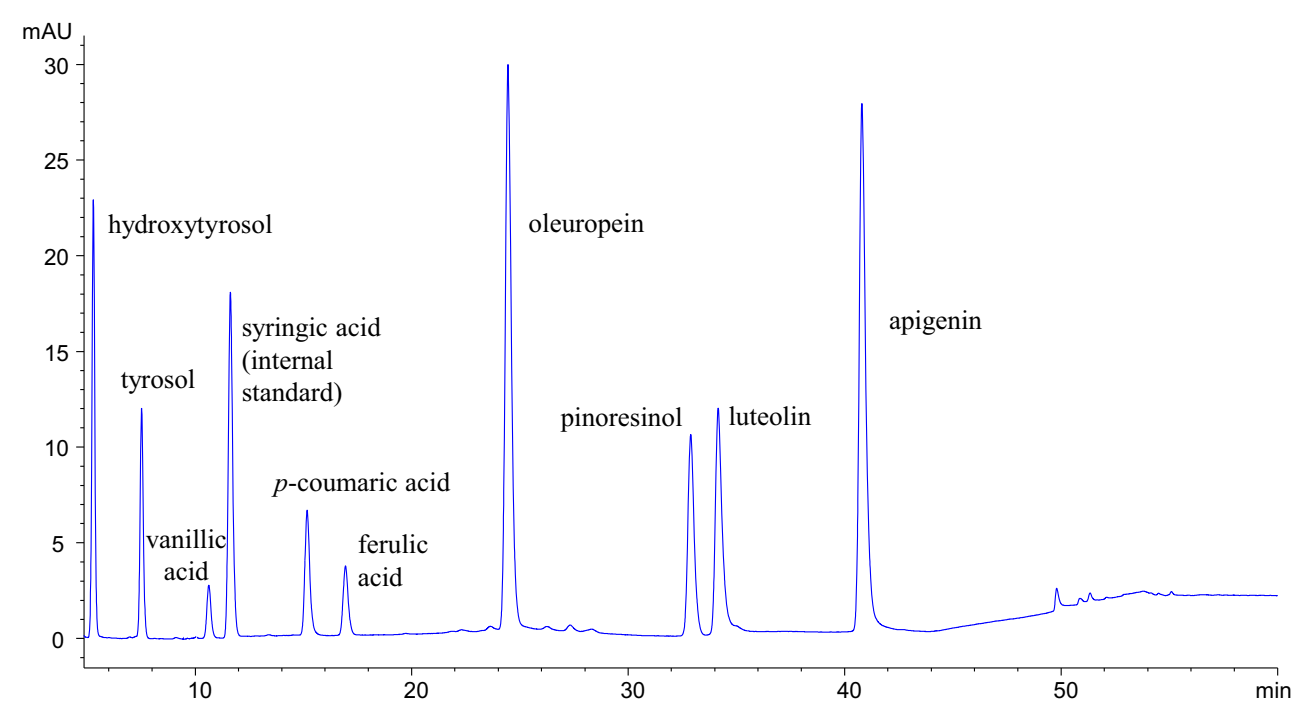

and based on our previous results (Ricciutelli et al. 2017). Due to the lack of the commercial availability of the specific secoiridoid derivatives found in higher amount in the oil, the use of standards different from them was made in many studies (e.g., Franco et al. 2014; Caporaso et al. 2015; De La Torre-Carbot et al. 2005; Alessandri et al. 2014), unless the isolation of the above mentioned secoiridoid species is performed (Suárez et al. 2008; Selvaggini et al. 2006). The other analytes (phenolic alcohols, phenolic acids, lignans, and flavonoids) were quantified in the present study at their specific wavelengths by using their specific calibration curves. The micro-scaled method was validated by determining LOD, LOQ, recovery, linearity, repeatability, and reproducibility (Table 1). Linearity was very similar to the reference method and sensitivity was comparable or, in several cases, improved, as compared to the reference method. In fact, LOQ range for phenolic alcohols is $0.025-0.028 \mathrm{mg} \mathrm{kg}^{-1}$ while it was $0.17-$ $0.32 \mathrm{mg} \mathrm{kg}^{-1}$ in the reference method; for phenolic acids, it is
$0.008-0.056 \mathrm{mg} \mathrm{kg}^{-1}$ and it was $0.033-0.061 \mathrm{mg} \mathrm{kg}^{-1}$ in the reference method; for pinoresinol, it is $0.1 \mathrm{mg} \mathrm{kg}^{-1}$ and it was $0.39 \mathrm{mg} \mathrm{kg}^{-1}$; for flavonoids, it is $0.044-0.077 \mathrm{mg} \mathrm{kg}^{-1}$ and it was $0.096-0.109 \mathrm{mg} \mathrm{kg}^{-1}$; for oleuropein, it is $0.35 \mathrm{mg} \mathrm{kg}^{-1}$ and it was $1.15 \mathrm{mg} \mathrm{kg}^{-1}$ in the reference method. For most of compounds, LODs and LOQs obtained are similar or lower than values reported in recent literature by using HPLC-DAD (e.g., Franco et al. 2014; De La Torre-Carbot et al. 2005; Selvaggini et al. 2006) or HPLC with fluorimetric detection (HPLC-FLD) (Selvaggini et al. 2006). For example, with respect to Selvaggini et al. (2006), who reported a very convenient method in terms of sample preparation, our method presents lower LOQs for hydroxytyrosol (14-fold), tyrosol (11fold), and pinoresinol (10-fold) as compared to the HPLCDAD method, and 3.4, 3.6, and 35-fold respectively, as compared to the HPLC-FLD method. For oleuropein, we obtained a LOQ of $350 \mu \mathrm{g} \mathrm{kg}^{-1}$ (or $700 \mu \mathrm{g} \mathrm{L}^{-1}$ if expressed in the analyzed solution), thus similar to the value obtained in the
Fig. 2 Overlaid HPLC-DAD chromatograms $(\lambda=280 \mathrm{~nm})$ obtained from an extra virgin olive oil hydro-alcoholic extract (blue) and from an olive oil hydro-alcoholic extract (red) by applying the optimized method. The phenolic substances and the main secoiridoid quantified at $280 \mathrm{~nm}$ are indicated $(3,4-$ DHPEA-EDA dialdehydic form of decarboxymethyl elenolic acid linked to hydroxytyrosol, $p$ HPEA-EDA dialdehydic form of decarboxymethylelenolic acid linked to tyrosol, 3,4-DHPEA-EA isomer of oleuropein aglycone, $p$ HPEA-EA ligstroside aglycon)

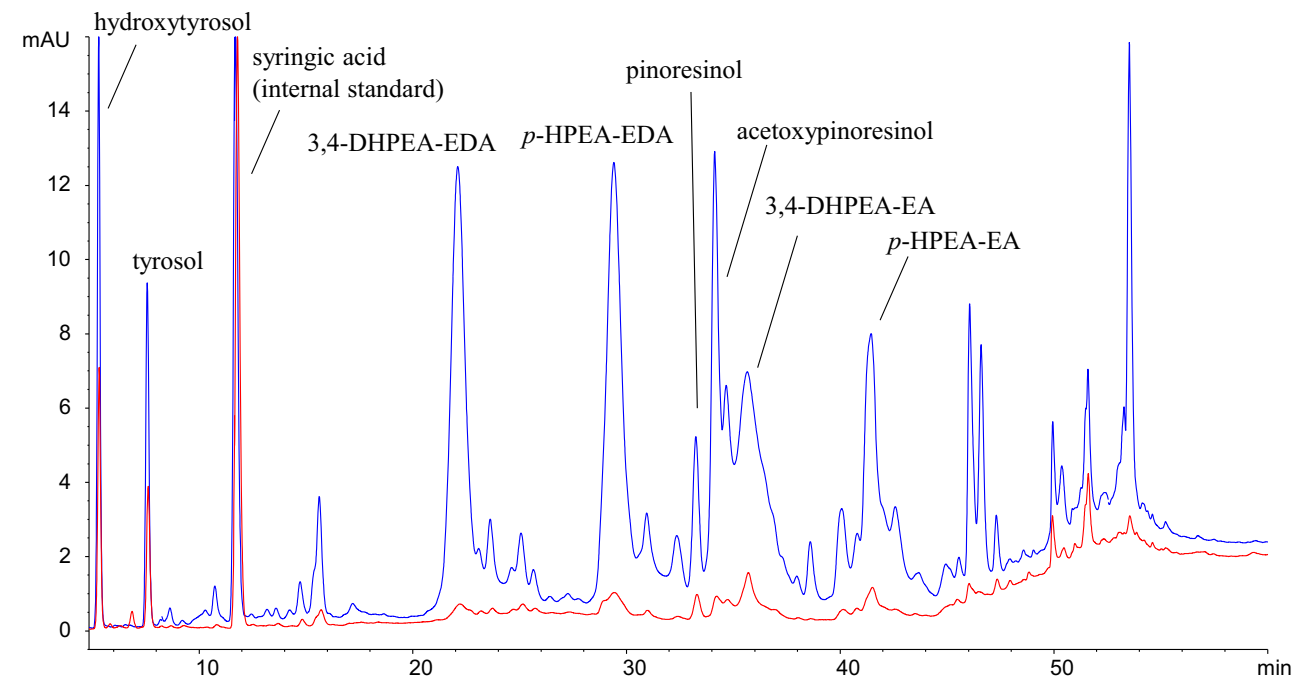




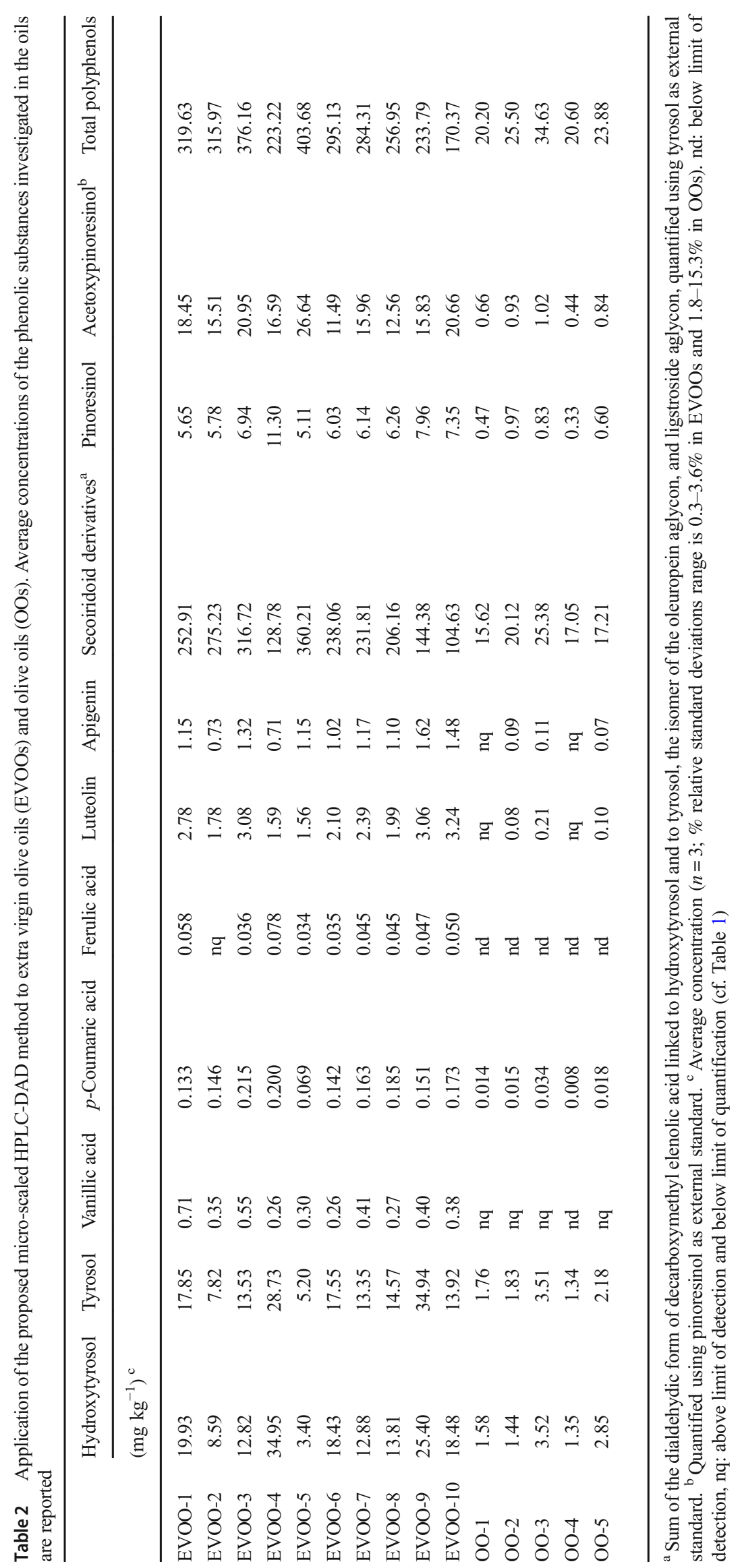


study of De La Torre-Carbot et al. (2005) $\left(600 \mu \mathrm{g} \mathrm{L}^{-1}\right)$. LOQ values found for tyrosol, $p$-coumaric acid, and for the flavonoids luteolin and apigenin are also slightly lower (7-, 2-, 2-, and 4-fold respectively) in comparison to those reported by De La Torre-Carbot et al. (2005). On the other side, LOQ found for vanillic acid in our study is 3-fold higher with respect to that reported by De La Torre-Carbot et al. (2005). Recovery, a parameter which could result more affected by the downscaled procedure due to both the lowered amounts of sample and solvents, as well as to the different glassware used, actually resulted to be only slightly lower as compared to the reference method (Ricciutelli et al. 2017) in which it ranged from 73 to $102 \%$; it remained in fact within values higher than $64 \%$, with a range of $64-97 \%$ for the first fortification level and of 70-100\% for the second fortification level, similarly to what obtained by Capriotti et al. (2014), in which it ranged from 75 to $105 \%$.

In the recovery experiments, the reproducibility of the method, in terms of \% RSD, was in the range $1.6-8.3 \%$ for the first fortification level and $1.2-4.1 \%$ for the second fortification level. Repeatability, in terms of RSD \%, was within the range $0.9-5.3 \%$ in the analysis of the analytical standard and $0.3-3.6 \%$ in the analysis of the sample; reproducibility gave comparable results to repeatability: $1.3-4.6 \%$ for the standard and $0.7-5.6 \%$ for the sample. Regarding sensitivity, it has been shown that it was widely sufficient to quantify all the analytes considered in all of the ten commercial EVOO samples investigated to which the method was applied. Instead, the quantification of the analytes was not always allowed in OOs due to the very low concentration of phenolic substances in this type of sample, where the refining process, in which the most part of the oil composing an $\mathrm{OO}$ is undergone, leads to a great reduction of the phenolic content.

The results obtained from the application of the method to 10 EVOOs and 5 OOs are reported in Table 2. In the EVOOs investigated, the total polyphenol content ranged from 170.37 to $403.68 \mathrm{mg} \mathrm{kg}^{-1}$; secoiridoids were found in the range $104.63-360.21 \mathrm{mg} \mathrm{kg}^{-1}$, free hydroxytyrosol and tyrosol at concentrations of $3.40-34.95 \mathrm{mg} \mathrm{kg}^{-1}$ and $5.20-$ $34.94 \mathrm{mg} \mathrm{kg}^{-1}$ respectively, phenolic acids at $0.41-$ $0.90 \mathrm{mg} \mathrm{kg}^{-1}$, lignans at $17.52-31.76 \mathrm{mg} \mathrm{kg}^{-1}$, and flavonoids at $2.31-4.72 \mathrm{mg} \mathrm{kg}^{-1}$. The results are in agreement with several other studies where the phenolic substances content in EVOOs is reported (e.g. Caporaso et al. 2015; Selvaggini et al. 2006; Alessandri et al. 2014; Servili et al. 2014). In the case of OOs, where the content of the hydrophilic phenolic compounds is much lower due to the refining process to which most part of $\mathrm{OO}$ is undergone, the proposed method does not allow the quantification of vanillic and ferulic acids, and sometimes of the flavonoids, while it is still adequate to quantify $p$-coumaric acid, free hydroxytyrosol and tyrosol (that are found in similar amount as compared to EVOOs), secoiridoid derivatives, and lignans, found in all the investigated samples in concentrations above LOQ values. In conclusion, the proposed micro-scaled method, while allowing the shortening of the working time and reducing solvent quantities, is still adequate for the quantification of phenolic substances in EVOO.

Funding This study was funded by the University of Camerino (grant "Fondo di Ateneo per la Ricerca" 2014-2015, project: “AEVOO: Authentication of Extra Virgin Olive Oil").

\section{Compliance with Ethical Standards}

Conflict of Interest Giovanni Caprioli declares that he has no conflict of interest. Maria Chiara Boarelli declares that she has no conflict of interest. Massimo Ricciutelli declares that he has no conflict of interest. Gianni Sagratini declares that he has no conflict of interest. Dennis Fiorini declares that she has no conflict of interest.

Ethical Approval This article does not contain any studies with human participants or animals performed by any of the authors.

Informed Consent Not applicable.

Publisher's Note Springer Nature remains neutral with regard to jurisdictional claims in published maps and institutional affiliations.

\section{References}

Alessandri S, Ieri F, Romani A (2014) Minor polar compounds in extra virgin olive oil: correlation between HPLC-DAD-MS and the FolinCiocalteu spectrophotometric method. J Agric Food Chem 62:826835

Bakhouche A, Lozano-Sanchez J, Beltran-Debon R, Joven J, SeguraCarretero A, Fernandez-Gutierrez A (2013) Phenolic characterization and geographical classification of commercial Arbequina extravirgin olive oils produced in southern Catalonia. Food Res Int 50: 401-408

Bayram B, Esatbeyoglu T, Schulze N, Ozcelik B, Frank J, Rimbach G (2012) Comprehensive analysis of polyphenols in 55 extra virgin olive oils by HPLC-ECD and their correlation with antioxidant activities. Plant Foods Hum Nutr 67:326-336

Bendini A, Cerretani L, Carrasco-Pancorbo A, Gómez-Caravaca AM, Segura-Carretero A, Fernández-Gutiérrez A, Lercker G (2007) Phenolic molecules in virgin olive oils: a survey of their sensory properties, health effects, antioxidant activity and analytical methods. An overview of the last decade. Molecules 12:1679-1719

Caporaso N, Savarese M, Paduano A, Guidone G, De Marco E, Sacchi R (2015) Nutritional quality assessment of extra virgin olive oil from the Italian retail market: do natural antioxidants satisfy EFSA health claims? J Food Compos Anal 40:154-162

Capriotti AL, Cavaliere C, Crescenzi C, Foglia P, Nascatelli R, Samperi R, Laganà A (2014) Comparison of extraction methods for the identification and quantification of polyphenols in virgin olive oil by ultra-HPLC-QtoF mass spectrometry. Food Chem 158:392-400

Carrasco-Pancorbo A, Cerretani L, Bendini A, Segura-Carretero A, Gallina-Toschi T, Fernandez-Gutiérrez A (2005) Analytical determination of polyphenols in olive oils. J Sep Sci 28:837-858

De La Torre-Carbot J, Gimeno C, Raventos LS (2005) Characterization and quantification of phenolic compounds in olive oils by solidphase extraction, HPLC-DAD, and HPLC-MS/MS. J Agric Food Chem 53:4331-4340 
EU (2012) Establishing a list of permitted health claims made on foods, other than those referring to the reduction of disease risk and to children's development and health. Commission Regulation (EU) 432/2012. Off J Eur Union 136:1-40

Flores MIA, Romero-González R, Frenich AG, Vidal JLM (2012) Analysis of phenolic compounds in olive oil by solid-phase extraction and ultra high performance liquid chromatography-tandem mass spectrometry. Food Chem 134:2465-2472

Franco MN, Galeano-Diaz T, Lopez O, Fernandez-Bolanos JG, Sanchez J, De MC, Gil MV, Martin-Vertedor D (2014) Phenolic compounds and antioxidant capacity of virgin olive oil. Food Chem 163:289 298

IOC (2009) Determination of biophenols in olive oils by HPLC. COI/ T.20/Doc. No 29/1-8

Montedoro GF, Servili M, Baldioli M, Miniati E (1992) Simple and hydrolyzable phenolic compounds in virgin olive oil. 1 . Their extraction, separation, and quantitative and semiquantitative evaluation by HPLC. J Agric Food Chem 40:1571-1576

Pirisi FM, Angioni A, Cabras P, Garau VL, Di Teulada MTS, Dos Santos MK, Bandino G (1997) Phenolic compounds in virgin olive oils. I. Low-wavelength quantitative determination of complex phenols by high-performance liquid chromatography under isocratic elution. J Chromatogr A 768:207-213

Pirisi FM, Cabras P, Falqui Cao C, Migliorini M, Muggelli M (2000) Phenolic compounds in virgin olive oil. 2. Reappraisal of the extraction, HPLC separation, and quantification procedures. J Agric Food Chem 48:1191-1196

Ricciutelli M, Marconi S, Boarelli MC, Caprioli G, Sagratini G, Ballini R, Fiorini D (2017) Olive oil polyphenols: a quantitative method by high-performance liquid-chromatography-diode-array detection for their determination and the assessment of the related health claim. J Chromatogr A 1481:53-63

Selvaggini R, Servili M, Urbani S, Esposto S, Taticchi A, Montedoro G (2006) Evaluation of phenolic compounds in virgin olive oil by direct injection in high-performance liquid chromatography with fluorimetric detection. J Agric Food Chem 54:2832-2838

Servili M, Sordini B, Esposto S, Urbani S, Veneziani G, Di Maio I, Selvaggini R, Taticchi A (2014) Biological activities of phenolic compounds of extra virgin olive oil. Antioxidants 3:1-23

Suárez M, Macià A, Romero MP, Motilva MJ (2008) Improved liquid chromatography tandem mass spectrometry method for the determination of phenolic compounds in virgin olive oil. J Chromatogr A 1214:90-99 